\title{
An Image Filter Based on Improved Shearlet Transformation
}

\author{
Qingfeng Xia ${ }^{1, a}$, Yan Zhang ${ }^{2, b}$, Shuting Zeng ${ }^{3, c}$ \\ ${ }^{1}$ Laboratory of Robotics, Nanjing University Jinling College, Nanjing, 210089, China \\ ${ }^{2}$ Laboratory of Robotics, Nanjing University Jinling College, Nanjing, 210089, China \\ ${ }^{3}$ Laboratory of Robotics, Nanjing University Jinling College, Nanjing, 210089, China \\ aemail: qingfeng_xia_j@163.com, bemail: 717252987@qq.com, 'email: shuting_42@163.com
}

Keywords: Shearlet transform; Image denoise; Fiter

\begin{abstract}
Image restoration processing is to rebuild or restore original images with some prior knowledge according to given degraded images or noise-polluted images. This paper proposed an image filter algorithm based on improved Shearlet Transformation. Firstly, it adopted classical Shearlet Transform to decompose noised image into many sub-wavelets under multi-scale and multi-orientation. Secondly, it weighted each sub-wavelets obtained in first step. Then, by classical Shearlet inverse Transform, a new composite image created which composed from those weighted sub-wavelets. Experimental results shown that proposed algorithm eliminates noise effectively and yields good Peak Signal Noise Ratio (PSNR).
\end{abstract}

\section{Introduction}

Image is one important environment sensing channel to machine, but on the processes of formation, transmission, reception, it always be contaminated by noise, and make following processes be difficult. So noise reduction becomes one of the most important problems in image processing.

So far, there are three mainly methods to do this job, there are transform domain method, spatial domain method and Partial Differential Equations(PDE) method. Recently, in transform domain, Professor Labate proposed Shearlet algorithm, which is a novel class of multi-dimensional representation systems. One advantage of this approach is that these systems can be constructed using generalized multi-resolution analysis and implemented efficiently using a classical cascade algorithm [1 8]. Due to Shearlet Transform have multi-scale and multi-direction feature, simple hard threshold denoising method could yield not bad performance in its practical application of image de-noise[9 14]. But in iteration[15 16], how to determine threshold rule was untouched, but it is one important parameter in this algorithm, and will affect result directly, so we hold this opinion that this last step is worth studying and the findings has theoretical value and practical directive meaning.

Here, in this paper, we intend to propose a new threshold rule, and hope it can improve algorithm's image denoise performance when calculation price is not increase. After many experiments, result data shown that proposed threshold can achieve satisfactory performance.

The remaining paper is organized as follows. Section 2 introduces related theories. Section 3 explains our algorithm, including workflow, section 4 presents the experiment results of proposed algorithm and Section 5 concludes this paper.

\section{Related Theories}

Labate etc[1,2] proposed Shearlet transform based on wavelet. In dimension $n=2$, affine system:

$$
\Psi_{A B}(\psi)=\left\{\psi_{j, l, k}(x)=|\operatorname{det} A|^{j / 2} \psi\left(B^{l} A^{j} x-k\right): j, l \in \mathbb{Z}, k \in \mathbb{Z}^{2}\right\}
$$

here, $\psi \in L^{2}\left(\mathbb{R}^{2}\right)$, A, B is $2 * 2$ invertible matrices with $|\operatorname{det} B|=1$. 
If $\Psi_{A B}(\psi)$ satisfied Parseval $L^{2}\left(\mathbb{R}^{2}\right)$, then, those elements of $\Psi_{A B}(\psi)$ are called composite wavelets.

Shearlet is a special example of $L^{2}\left(\mathbb{R}^{2}\right)$, for only when

$$
A=A_{0}=\left(\begin{array}{ll}
4 & 0 \\
0 & 2
\end{array}\right), B=B_{0}=\left(\begin{array}{ll}
0 & 1 \\
1 & 1
\end{array}\right),
$$

here $A=A_{0}$ is the anisotropic dilation matrix, $B=B_{0}$ is the shear matrix.

For $\xi=\left(\xi_{1}, \xi_{2}\right) \in \hat{\mathbb{R}}^{2}, \xi_{1} \neq 0$, when $\psi^{(0)}, \hat{\psi}_{1}, \hat{\psi}_{2}$ satisfy

$$
\left\{\begin{array}{l}
\hat{\psi}^{(0)}(\xi)=\hat{\psi}^{(0)}\left(\xi_{1}, \xi_{2}\right)=\hat{\psi}_{1}\left(\xi_{1}\right) \hat{\psi}_{2}\left(\xi_{2} / \xi_{1}\right) \\
\hat{\psi}_{1}, \hat{\psi}_{2} \in C^{\infty}(\hat{\mathbb{R}}), \operatorname{supp} \hat{\psi}_{1} \subset[-1 / 2,-1 / 16] \cup[1 / 16,1 / 2], \operatorname{supp} \hat{\psi}_{2} \subset[-1,1] \\
\sum_{j \geq 0}\left|\hat{\psi}_{1}\left(2^{-2 j} \omega\right)\right|^{2}=1 \text { for }|\omega| \geq 1 / 8, j \geq 0 \\
\sum_{l=-2^{j}}^{2^{j}-1}\left|\hat{\psi}_{2}\left(2^{j} \omega-l\right)\right|^{2}=1 \text { for }|\omega| \leq 1
\end{array}\right.
$$

Then, we get

$$
\sum_{j \geq 0} \sum_{l=-2^{j}}^{2^{j}-1}\left|\hat{\psi}^{(0)}\left(\xi A_{0}^{-j} B_{0}^{-l}\right)\right|^{2}=\sum_{j \geq 0} \sum_{l=-2^{j}}^{2^{j}-1}\left|\hat{\psi}_{1}\left(2^{-2 j} \xi_{1}\right)\right|^{2}\left|\hat{\psi}_{2}\left(2^{j} \xi_{2} / \xi_{1}-l\right)\right|^{2}=1
$$

Then, $\left\{\hat{\psi}^{(0)}\left(\xi A_{0}^{-j} B_{0}^{-l}\right)\right\}$ form a tiling of the set

$$
D_{0}=\left\{\left(\xi_{1}, \xi_{2}\right) \in \hat{\mathbb{R}}^{2}:\left|\xi_{1}\right| \geq 1 / 8,\left|\xi_{2} / \xi_{1}\right| \leq 1\right\}
$$

From the condition on the support of $\hat{\psi}_{1}, \hat{\psi}_{2}$, it is easily deduced that $\hat{\psi}_{j, l, k}$ have frequency support contained in the set.

$\operatorname{supp} \hat{\psi}_{j, l, k}^{(0)} \subset\left\{\left(\xi_{1}, \xi_{2}\right): \xi_{1} \in\left[-2^{2 j-1},-2^{2 j-4}\right] \cup\left[2^{2 j-4}, 2^{2 j-1}\right],\left|\xi_{2} / \xi_{1}+l 2^{-2 j}\right| \leq 2^{-j}\right\}$, Thus, every element in $\psi_{j, l, k}$ is supported on a pair of trapezoids of approximate size $2^{2 j} \times 2^{j}$, oriented along lines of slope $12^{-j}$.

For $L^{2}\left(D_{1}\right)^{\vee}$, here $D_{1}$ is the vertical cone, when formula (4) was satisfied:

$$
\left\{\begin{array}{l}
D_{1}=\left\{\left(\xi_{1}, \xi_{2}\right) \in \hat{\mathbb{R}}^{2}:\left|\xi_{2}\right| \geq 1 / 8,\left|\xi_{1} / \xi_{2}\right| \leq 1\right\} \\
A_{1}=\left(\begin{array}{ll}
2 & 0 \\
0 & 4
\end{array}\right), B_{1}=\left(\begin{array}{ll}
1 & 0 \\
1 & 1
\end{array}\right) \\
\hat{\psi}^{(1)}(\xi)=\hat{\psi}^{(1)}\left(\xi_{1}, \xi_{2}\right)=\hat{\psi}_{1}\left(\xi_{2}\right) \hat{\psi}_{2}\left(\xi_{1} / \xi_{2}\right)
\end{array}\right.
$$

Then, collection $\left\{\psi_{j, l, k}^{(1)}(x)=2^{3 j / 2} \psi^{(1)}\left(B_{1}^{l} A_{1}^{j} x-k\right): j \geq 0,-2^{j} \leq l \leq 2^{j}-1, k \in \mathbb{Z}^{2}\right\}$ is a Parseval frame for $L^{2}\left(D_{1}\right)^{\vee}$.

\section{Threshold rule}

Threshold rule is the most important problem in image denoising of transform domain. Generally speaking, there are two kinds of threshold rule, the hard-threshold and the soft-threshold. Donoho[15 16] proposed a threshold rule: 


$$
\delta=\sigma \sqrt{2 \ln (N)}
$$

here, $\mathrm{N}$ pixels number of image, $\sigma$ is noise level.

There are lots of literatures shows that Donoho threshold is not the optimal threshold. Here, we proposed a soft threshold rule.

The Sigmoid curve is a mathematical concept which has been widely used to model the natural life cycle of many things. A sigmoid function is a mathematical function having an " $\mathrm{S}$ " shape (sigmoid curve). Often, sigmoid function refers to the special case of the logistic function shown in the first figure and defined by the formula. A wide variety of sigmoid functions have been used as the activation function of artificial neurons, including the logistic and hyperbolic tangent functions. Sigmoid curves are also common in statistics as cumulative distribution functions, such as the integrals of the logistic distribution, the normal distribution, and Student's probability density functions. So ,here we use it as a part of our soft threshold rule.

$$
\begin{aligned}
& \delta_{k, j}=\operatorname{Sigmoid}(\sigma \sqrt{2 \ln (N)}) \\
& \text { Sigmoid }=\frac{1}{1+e^{-v}}
\end{aligned}
$$

Classical value function (5) is proved that it is upper bounds of rule, and from function(6), we can see that, new soft threshold value is smaller than classical value, it means that new soft threshold value is within the upper bound, it should has better performance. From another perspective, new rule adopts sigmoid function as soft threshold; this new function will suitable for mankind's Neural Network (NN) optical implementation.

\section{Workflow}

Just like classical Shearlet algorithm[1 2,4 9], our proposed image denoise algorithm divided into 3 steps:

Step 1. It adopted classical Shearlet Transform to decompose noised image into many sub-wavelets under multi-scale and multi-orientation.

Step 2. It uses new threshold rule to determine each sub-wavelets.

Step 3. By classical Shearlet inverse Transform, a new composite image created which composed from those weighted sub-wavelets.

\section{Experimental Results}

Here, we used two classical images to test our algorithm, Fig1 (a) is Barbara and Fig1 (d) is Baboon. Added 30\% salt and pepper noise into them, got Fig1 (b) and Fig1 (e). Then, we adopted our proposed algorithm to recovery them, after calculation, those results were Fig1(c) and Fig1 (f). From Fig1 we can see that, our algorithm can recovery noised image with satisfactory performance, and that is our research purpose. 


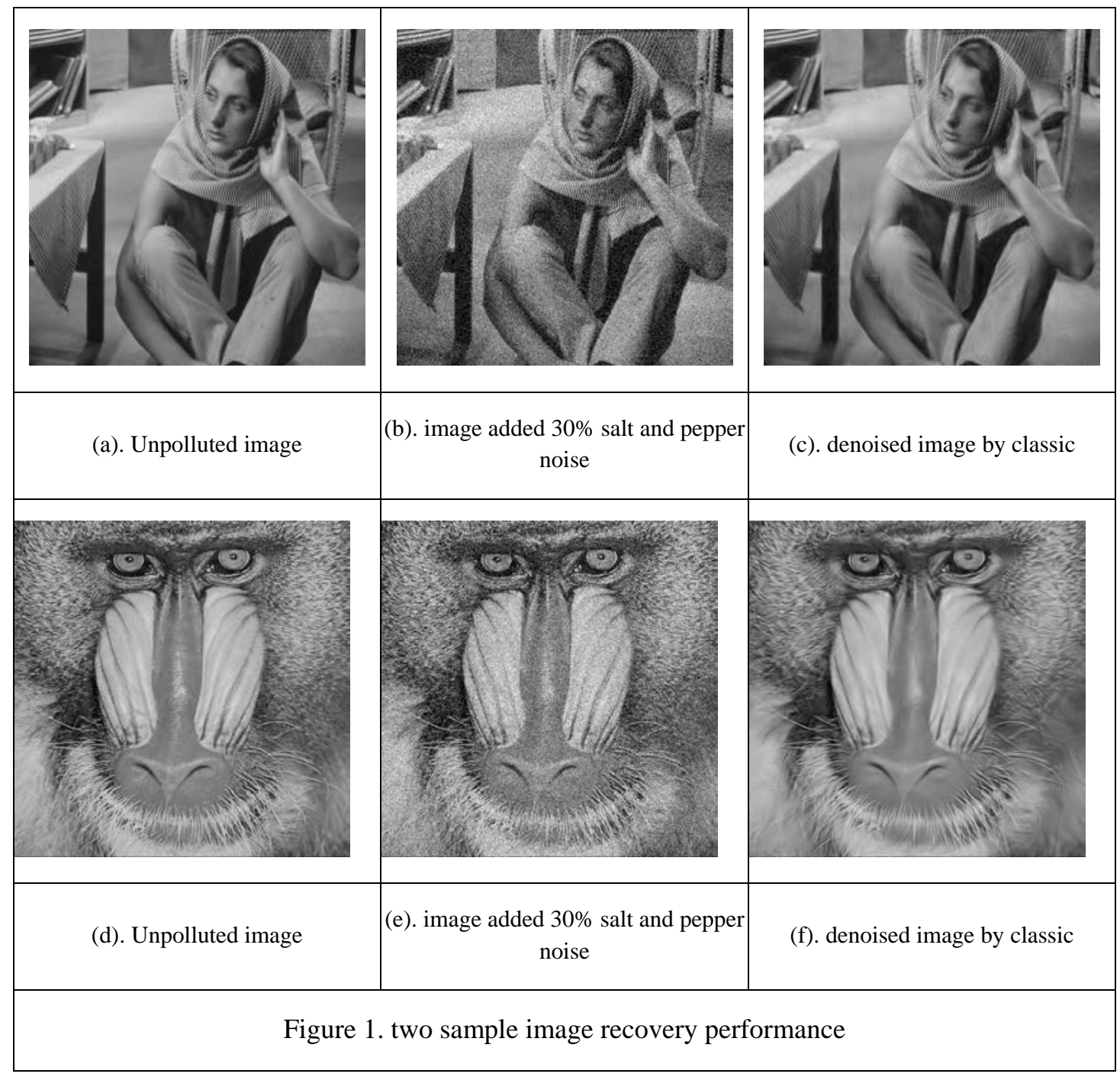

\section{Conclusion}

In this paper, we intend to propose a new threshold rule to Shearlet Transform algorithm, and hope it can improve algorithm's image denoise performance when calculation price is not increase. After many experiments, result data shown that proposed threshold can achieve satisfactory performance.

\section{Acknowledgements}

In this paper, the research was sponsored by the Project of 2014 Nanjing University Jinling College Teaching Reform and Research (Project No. 0010521508).

\section{References}

[1] D. Labate, W. Lim, G. Kutyniok and G. Weiss, and "Sparse multidimensional representation using shearlets", Wavelets XI (San Diego, CA, 2005), 254-262, SPIE Proc. 5914 , SPIE, Bellingham, WA, 2005.

[2] D. Labate, and G. Weiss, "Wavelets associated with composite dilations", in: Matematicas: investigacion y educacion. Un homenaje a Miguel de Guzman. Universidad Complutense de Madrid, Ed.Anaya, Madrid, 2005.

[3] $\mathrm{Hu} \mathrm{K}$, Song A, Xia $\mathrm{M}$, et al. An adaptive filtering algorithm based on genetic algorithm-backpropagation network[J]. Mathematical Problems in Engineering, 2013, 2013. 
[4] Labate D, Lim W Q, Kutyniok G, et al. Sparse multidimensional representation using shearlets[C]//Optics \& Photonics 2005. International Society for Optics and Photonics, 2005: 59140U-59140U-9.

[5] Yi S, Labate D, Easley G R, et al. Edge detection and processing using shearlets[C]//mage Processing, 2008. ICIP 2008. 15th IEEE International Conference on. IEEE, 2008: 1148-1151.

[6] Easley, G.R.; Labate, D.; Colonna, F., "Shearlet-Based Total Variation Diffusion for Denoising," Image Processing, IEEE Transactions on , vol.18, no.2, pp.260,268, Feb. 2009.

[7] G. R. Easley, D. Labate and V. Patel, "Directional multiscale processing of images using wavelets with composite dilations", J. Math. Imag. Vision 48(1) p. 13-34 (2014).

[8] Zhi-yong Fan, Quan-sen Sun, Ze-xuan Ji, Kai Hu. An Image Filter Based on Multiobjective Genetic Algorithm and Shearlet Transformation. Mathematical Problems in Engineering.2013,463760.

[9] Zhiyong Fan etc. An Improved Image Denoising Algorithm based on Shearlet. International Journal of Signal Processing, Image Processing and Pattern Recognition. Vol. 6, No. 4, August, 2013.

[10] Min Xia, W.K. Wong, A seasonal discrete grey forecasting model for fashion retailing,Knowledge-Based Systems, 57, pp. 119-126, 2014.

[11] Hu K, Song A G, Zhang Y C, et al. Delay-range-dependent Stability Criteria of Neural Networks with Time-varying Discrete and Distributed Delays[J]. Int J Adv Robot Syst, 2014, 11: 23.

[12] Hu K, Song A G, Wang W L, et al. Fault detection and estimation for non-Gaussian stochastic systems with time varying delay[J]. Advances in Difference Equations, 2013, 2013(1): 1-12.

[13] Min Xia, Yingchao Zhang, Liguo Weng, Xiaoling Ye, Fashion retailing forecasting based on extreme learning machine with adaptive metrics of inputs, Knowledge-Based Systems 36 (2012) 253-259.

[14] Song A, Han Y, Hu H, et al. Active perception-based haptic texture sensor[J]. Sensors and Materials, 2013, 25(1): 1-15.

[15] Min Xia, Zhijie Wang, Jian'an Fang, Temporal association based on dynamic depression synapses and chaotic neurons, Neurocomputing, 74 (2011) 3242-3247.

[16] Min Xia, Liguo Weng, Xiaoling Ye, Sequence Memory based on Ordered Pattern Interrelation, Advanced science letters, Volume 5, Number 2, February 2012 , pp. 547-551(5). 